\title{
Performance Analysis of Surface Condenser in 525MW Thermal Power Plant
}

\author{
Gaurav Masiwal $^{\# 1}$, P.S.Kumar ${ }^{* 2}$, Sumit Chaudhary ${ }^{* 3}$ \\ \# Operation and Efficiency Department, \\ Steag Operation and Maintenance Company Private Limited (SOMC), Andhra Pradesh, India \\ ${ }^{1}$ masiwalgaurav@gmail.com \\ * Power Plant Professional, \\ Steag Operation and Maintenance Company Private Limited (SOMC), Andhra Pradesh, India \\ 2 ps.kumar@somc.co.in \\ * Mechanical Department, Delhi Technological University, Delhi, India \\ ${ }^{3}$ mait.sumit@gmail.com
}

\begin{abstract}
This paper presents off design performance evaluation and calculation methodology of a surface type condenser. Best condenser pressure which can be achieved in actual off design conditions has been evaluated by real time parameters. Condenser performance study has been carried out for cooling water flow, cooling water inlet temperature, and for air ingress/dirty tubes. This method can be proved useful in the case where no curve regarding variation of condenser back pressure verses cooling water inlet temperature is available. All data for performance has been collected and evaluated from a 525MW operating unit of Bharat Heavy Electricals Limited.
\end{abstract}

Keyword - Surface Condenser; Heat Transfer; Steam Condenser; Heat Load; Condenser performance; Power Plant

\section{INTRODUCTION}

This paper is based on performance analysis of condenser in a 525MW thermal power plant. A thermal power plant consists of five major components - (1) boiler, (2) steam turbines - high pressure turbine, intermediate pressure turbine and low pressure turbine, (3) condenser, (4) feed water pumps - condensate extraction pumps and boiler feed pumps, (5) feed water heaters - one feed water heater (steam bled from high pressure turbine exhaust), one feed water heater (steam bled from intermediate pressure turbine) and three feed water heater for which steam is bled from low pressure turbine [1]. In boiler, constant pressure heating of feed water takes place in economizer, evaporator and in superheater. Superheated steam then goes to high pressure turbine, expands to produce work. Some part of steam is extracted here for feed water heating process. Steam then is reheated to high temperature and enters in intermediate pressure turbine. Some steam is again bled for regeneration process. Steam then enters in low pressure turbine where again some portion of steam is extracted for feed water heating process. After passing through the low pressure turbine, steam goes to condenser where this steam converts into saturated water. Low pressure feed water pumps (condensate extraction pumps) pump condensate water to the low pressure heaters where this condensate water gets some heat from extracted steam. After low pressure heaters, condensate water goes to Deaerator and then with the help of high pressure feed water pumps (boiler feed pumps) feed water enters the boiler via high pressure heaters. And this way whole cycle repeats itself. Condenser is an essential component in thermal power plant. It is a type of heat exchanger in which steam undergoes phase change by giving latent heat and converts into water. In turn coolant water gains sensible heat. As specific volume of steam is more than that of specific volume of condensed water. A vacuum (negative pressure) develops in shell side of condenser which extracts more steam from low pressure turbine exhaust and thus creates a self suction natural phenomenon. Condenser reduces the turbine exhaust pressure so as to increase the specific output of turbine. Vikram Haldkar, Abhay kumar sharma, R.K Ranjan, and V.K Bajpai [5] have worked on performance analysis of a surface type condenser. Rafal Laskowski [6] has analysed steam condenser performance in off-design conditions as the function of inlet parameters. Sami I. Attia [7] has analyzed the influence of condenser cooling water temperature on the thermal efficiency of a nuclear power plant. R.K.Kapooria, S Kumar and K S kasana [8] did technological investigations and efficiency analysis of a steam heat exchange condenser. Ajeet Singh Sikarwar, Devendra Dandotiya and Surendra Kumar Agrawal [9] did performance analysis of a surface condenser under various operating parameters. Amir vosough, Alireza falahat, Sadegh vosough, Hasan nasr esfehani, Azam behjat and Roya naseri rad [10] has worked on improvement on power plant efficiency. Said M.A. Ibrahim and Sami I. Attia [11] have analyzed influence of condenser cooling seawater salinity changes on the thermal performance of a nuclear power plant. 
The objective of this paper is to analyze the condenser performance by comparing the actual condenser pressure with calculated condenser pressure. Deviation between actual and calculated condenser pressure has been break-up into three factors- (1) variation due to cooling water inlet temperature, (2) variation due to cooling water flow and (3) variation due to air ingress/dirty tubes. For calculating theoretical condenser pressure heat balance approach across condenser has been used. Net heat load on condenser has been evaluated by considering significant heat loads points.

\section{PLANT DESCRIPTION}

The power plant has a total installed power capacity of 1050MW. The power house consists of two steam turbine unit of 525MW each. The schematic diagram of one 525MW unit is shown in Fig.1. This unit employs reheating and regenerative feed water heating system. Feed water heating is carried out in two stages of high pressure heaters (HPH-6, HPH-5) and three stages of low pressure heaters (LPH-3, LPH-2, LPH-1) along with one deaerating heat exchanger. Steam is superheated to 170 bar and $537^{\circ} \mathrm{C}$ in the steam generator and fed to the high pressure turbine. The high pressure turbine exhaust then goes to reheater where steam is reheated to $537{ }^{\circ} \mathrm{C}$ and enters to intermediate pressure turbine. Low pressure turbine exhaust is sent to condenser. Condensate collected in hotwell is extracted and pumped by condensate extraction pumps (CEP) to the low pressure heaters. Feed water after LPH-3 goes to deaerator and then pumped by boiler feed pumps to the high pressure heaters. And thus whole cycle repeats again. Single pass, Surface type shell and tube condenser manufactured by BHEL is provided in system. Condenser cooling medium is sea water. For prevention against corrosion and erosion, titanium tubes are provided. Sodium hypochlorite dosing is done at cooling water pump suction to avoid aqua culture and biological growth. Operating conditions of the power plant at $100 \%$ load is shown in Table I. Design details of condenser are shown in Table II.

\section{A. Nomenclature}

\begin{tabular}{|c|c|}
\hline $\mathrm{M}_{\mathrm{c}}$ & Cooling water mass flow rate \\
\hline $\mathrm{C}$ & Specific heat capacity of cooling water \\
\hline CW & Cooling water \\
\hline $\mathrm{T}_{\mathrm{co}}$ & Cooling water exit temperature \\
\hline $\mathrm{T}_{\mathrm{ci}}$ & Cooling water inlet temperature \\
\hline$\Delta \mathrm{T}$ & Cooling water temperature rise \\
\hline $\mathrm{U}_{\text {uncorrected }}$ & Uncorrected overall heat transfer coefficient \\
\hline $\mathrm{U}_{\text {corrected }}$ & Corrected overall heat transfer coefficient \\
\hline $\mathrm{A}_{\text {surface }}$ & Heat transfer surface area of condenser \\
\hline LMTD & Logarithmic mean temperature difference \\
\hline TTD & Terminal temperature difference \\
\hline C.F ${ }_{\mathrm{cw}}$, inlet temp & Correction factor for cooling water inlet temperature \\
\hline C.F tube material & Correction factor for condenser tube material \\
\hline C.F cleanliness factor & Correction factor for fouling \\
\hline $\mathrm{T}_{\text {sat }}$ & Saturation temperature of steam \\
\hline $\mathrm{P}_{\text {sat }}$ & Saturation pressure of steam \\
\hline $\mathrm{H}$ & Enthalpy \\
\hline MS & Main steam \\
\hline FW & Feed water \\
\hline BFP & Boiler feed pump \\
\hline $\mathrm{SH}$ & Superheater \\
\hline $\mathrm{RH}$ & Reheater \\
\hline S.C & Stator current \\
\hline $\mathrm{CRH}$ & HP turbine exhaust \\
\hline $\mathrm{HRH}$ & Reheated steam \\
\hline$P_{\text {gen }}$ & Power generation \\
\hline TDBFP & Turbine driven boiler feed pump \\
\hline
\end{tabular}






Fig.1. Schematic diagram of power plant.

Table I. Design Conditions of Power Plant at 100\% Load with 0\% Make Up

\begin{tabular}{|c|l|c|}
\hline S.No & Operating Condition at 100\% Load (0\% make up) & Value \\
\hline 1 & Power output $(\mathrm{MW})$ & 525 \\
\hline 2 & Main steam flow $(\mathrm{kg} / \mathrm{s})$ & 436 \\
\hline 3 & Main steam pressure (bar) & 170 \\
\hline 4 & Main steam temperature $\left({ }^{\circ} \mathrm{C}\right)$ & 537 \\
\hline 5 & Reheater flow $(\mathrm{kg} / \mathrm{s})$ & 390 \\
\hline 6 & Reheated steam pressure $($ bar $)$ & 40.43 \\
\hline 7 & Reheated steam temperature $\left({ }^{\circ} \mathrm{C}\right)$ & 537 \\
\hline 8 & Feed water flow $(\mathrm{kg} / \mathrm{s})$ & 436 \\
\hline 9 & Feed water inlet temperature to boiler $\left({ }^{\circ} \mathrm{C}\right)$ & 253.4 \\
\hline
\end{tabular}

Table II. Design Details of Condenser

\begin{tabular}{|c|l|c|}
\hline S.No & Design Details & Value \\
\hline 1 & Number of passes & 1 \\
\hline 2 & Cooling water flow $\left(\mathrm{m}^{3} / \mathrm{s}\right)$ & 24 \\
\hline 3 & Surface area of condenser $\left(\mathrm{m}^{2}\right)$ & 20627.4 \\
\hline 4 & Cooling water inlet temperature $\left({ }^{\circ} \mathrm{C}\right)$ & 32 \\
\hline 5 & Cooling water temperature rise $\left({ }^{\circ} \mathrm{C}\right)$ & 7 \\
\hline 6 & Terminal temperature difference $(\mathrm{TTD})-\left({ }^{\circ} \mathrm{C}\right)$ & 6.46 \\
\hline 7 & Log mean temperature difference $(\mathrm{LMTD})-\left({ }^{\circ} \mathrm{C}\right)$ & 9.5 \\
\hline 8 & Specific heat capacity of sea water- $\left(\mathrm{kJ} / \mathrm{kg}-{ }^{\circ} \mathrm{C}\right)$ & 3.883 \\
\hline 9 & Density of sea cooling water $\left(\mathrm{kg} / \mathrm{m}^{3}\right)$ & 1047 \\
\hline 10 & Cleanliness factor & 0.9 \\
\hline 11 & Condenser pressure $($ bar $)$ & 0.098 \\
\hline
\end{tabular}




\section{III.CALCULATION METHODOLOGY}

Applying first law of thermodynamics, energy balance for the condenser can be made.

Net heat load on condenser $=$ Heat gained by cooling water.

Net heat load on condenser $=\mathrm{M}_{\mathrm{c}} * \mathrm{C} *\left(\mathrm{~T}_{\mathrm{co}}-\mathrm{T}_{\mathrm{ci}}\right)$

The heat load on condenser is from the following sources: - Heat added to main steam, heat added in reheating, heat addition to feed water due to friction in boiler feed pump, heat addition due to superheater spray, heat addition due to reheater spray. Governing equation for heat addition due to different sources is given in Table III. Net heat load on condenser at different loading conditions is shown in Table IV.

Net heat load on condenser $=\mathrm{U}_{\text {corrected }} * \mathrm{~A}_{\text {surface }} *$ LMTD

$\mathrm{U}_{\text {corrected }}=\mathrm{U}_{\text {uncorrected }} * \mathrm{C} . \mathrm{F}_{\mathrm{cw} \text {, inlet temp }} * \mathrm{C} . \mathrm{F}_{\text {tube material }} *$ C.F cleanliness factor. [2]

Table III. Equations for Calculation of Net Heat Load on Condenser

\begin{tabular}{|c|l|l|}
\hline S.No & Parameters & \multicolumn{1}{|c|}{ Equations } \\
\hline 1 & Heat added by main steam $(\mathrm{kJ} / \mathrm{s})$ & $\mathrm{H}_{\mathrm{MS}}=\mathrm{m}_{\mathrm{ms}} *\left(\mathrm{~h}_{\mathrm{MS}}-\mathrm{h}_{\mathrm{FW}}\right)$ \\
\hline 2 & Heat added by reheated steam $(\mathrm{kJ} / \mathrm{s})$ & $\mathrm{H}_{\mathrm{HRH}}=\mathrm{m}_{\mathrm{hrh}} *\left(\mathrm{~h}_{\mathrm{HRH}}-\mathrm{h}_{\mathrm{CRH}}\right)$ \\
\hline 3 & $\begin{array}{l}\text { Heat added by boiler feed pump } \\
(\mathrm{kJ} / \mathrm{s})\end{array}$ & $\mathrm{H}_{\mathrm{BFP}}=\mathrm{m}_{\mathrm{fw}} *\left(\mathrm{~h}_{\mathrm{BFP}, \text { out }}-\mathrm{h}_{\mathrm{BFP}, \text { in }}\right)$ \\
\hline 4 & $\begin{array}{l}\text { Heat added by superheater spray } \\
(\mathrm{kJ} / \mathrm{s})\end{array}$ & $\mathrm{H}_{\mathrm{SH} \text { spray }}=\mathrm{m}_{\mathrm{SH} \text { spray }} *\left(\mathrm{~h}_{\mathrm{MS}}-\mathrm{h}_{\mathrm{SH} \text { spray }}\right)$ \\
\hline 5 & Heat added by reheater spray $(\mathrm{kJ} / \mathrm{s})$ & $\mathrm{H}_{\mathrm{RH} \text { spray }}=\mathrm{m}_{\mathrm{RH} \text { spray }} *\left(\mathrm{~h}_{\mathrm{HRH}}-\mathrm{h}_{\mathrm{RH} \text { spray }}\right)$ \\
\hline 6 & Stator current losses $(\mathrm{kJ} / \mathrm{s})$ & $\mathrm{H}_{\mathrm{S} . \mathrm{C}}=\left(\mathrm{I}_{\mathrm{armature}}\right)^{2} * \mathrm{R}_{\text {armature }}$ \\
\hline 7 & Power generation losses $(\mathrm{kJ} / \mathrm{s})$ & $\mathrm{P}_{\text {gen loss }}=0.1 \% \mathrm{P}_{\text {gen }}+\mathrm{H}_{\text {mech loss }}+\mathrm{H}_{\text {iron loss }}+\mathrm{H}_{\text {stator loss }}$ \\
\hline 8 & Net heat load on condenser $(\mathrm{kJ} / \mathrm{s})$ & $\begin{array}{l}\mathrm{H}_{\text {net load }}=\mathrm{H}_{\mathrm{MS}}+\mathrm{H}_{\mathrm{HRH}}+\mathrm{H}_{\mathrm{BFP}}+\mathrm{H}_{\mathrm{SH} \text { spray }}+\mathrm{H}_{\mathrm{RH} \text { spray }}-\mathrm{P}_{\text {gen }}-\mathrm{P}_{\text {gen }} \\
\text { loss. }\end{array}$ \\
\hline
\end{tabular}

Saturation temperature $\left(\mathrm{T}_{\text {sat }}\right)$ can be derived from Log mean temperature difference expression in the following manner. Temperature profiles of hot and cold fluids are shown in Fig. 2. in which steam is condensing at saturation temperature $\left(\mathrm{T}_{\text {sat }}\right)$ and cooling water enters at $\mathrm{T}_{\mathrm{ci}}$ and exit at $\mathrm{T}_{\mathrm{co}}$.

$$
\begin{aligned}
& \operatorname{LMTD}=\left(\Delta \mathrm{T}_{\mathrm{i}}-\Delta \mathrm{T}_{\mathrm{o}}\right) / \operatorname{Ln}\left(\Delta \mathrm{T}_{\mathrm{i}} / \Delta \mathrm{T}_{\mathrm{o}}\right) \\
& \Delta \mathrm{T}_{\mathrm{i}}=\mathrm{T}_{\mathrm{sat}}-\mathrm{Tc}_{\mathrm{i}} \\
& \Delta \mathrm{T}_{\mathrm{o}}=\mathrm{T}_{\mathrm{sat}}-\mathrm{Tc}_{\mathrm{o}} \\
& \mathrm{T}_{\mathrm{co}}-\mathrm{T}_{\mathrm{ci}}=\Delta \mathrm{T} \\
& \mathrm{LMTD}=\left[\left(\mathrm{T}_{\mathrm{sat}}-\mathrm{T}_{\mathrm{ci}}\right)-\left(\mathrm{T}_{\mathrm{sat}}-\mathrm{T}_{\mathrm{co}}\right)\right] / \operatorname{Ln}\left[\left(\mathrm{Ts}_{\mathrm{at}}-\mathrm{T}_{\mathrm{ci}}\right) /\left(\mathrm{T}_{\mathrm{sat}}-\mathrm{T}_{\mathrm{co}}\right)\right] \\
& \mathrm{LMTD}=\left[\mathrm{T}_{\text {sat }}-\mathrm{Tc}_{\mathrm{i}}-\mathrm{T}_{\text {sat }}+\mathrm{T}_{\mathrm{co}}\right] / \operatorname{Ln}\left[\left(\mathrm{T}_{\mathrm{sat}}-\mathrm{T}_{\mathrm{ci}}\right) /\left(\mathrm{T}_{\mathrm{sat}}-\mathrm{T}_{\mathrm{co}}\right)\right] \\
& \operatorname{LMTD}=\left[\mathrm{T}_{\mathrm{co}}-\mathrm{T}_{\mathrm{ci}}\right] / \operatorname{Ln}\left[\left(\mathrm{T}_{\mathrm{sat}}-\mathrm{T}_{\mathrm{ci}}\right) /\left(\mathrm{T}_{\mathrm{sat}}-\mathrm{T}_{\mathrm{co}}\right)\right] \\
& \mathrm{LMTD}=\Delta \mathrm{T} / \operatorname{Ln}\left[\left(\mathrm{T}_{\mathrm{sat}}-\mathrm{T}_{\mathrm{ci}}\right) /\left(\mathrm{T}_{\mathrm{sat}}-\mathrm{T}_{\mathrm{co}}\right)\right] \\
& \operatorname{Ln}\left[\left(\mathrm{T}_{\text {sat }}-\mathrm{T}_{\mathrm{ci}}\right) /\left(\mathrm{T}_{\mathrm{sat}}-\mathrm{T}_{\mathrm{co}}\right)\right]=\Delta \mathrm{T} / \mathrm{LMTD} \\
& \text { Let } \Delta \mathrm{T} / \mathrm{LMTD}=\mathrm{X} \\
& \operatorname{Ln}\left[\left(\mathrm{T}_{\text {sat }}-\mathrm{T}_{\text {ci }}\right) /\left(\mathrm{T}_{\text {sat }}-\mathrm{T}_{\text {co }}\right)\right]=\mathrm{X} \\
& {\left[\left(\mathrm{T}_{\text {sat }}-\mathrm{T}_{\mathrm{ci}}\right) /\left(\mathrm{T}_{\mathrm{sat}}-\mathrm{T}_{\mathrm{co}}\right)\right]=\mathrm{e}^{\mathrm{x}}} \\
& \mathrm{T}_{\text {sat }}-\mathrm{T}_{\mathrm{ci}}=\mathrm{e}^{\mathrm{x}} *\left(\mathrm{~T}_{\mathrm{sat}}-\mathrm{T}_{\mathrm{co}}\right) \\
& \mathrm{T}_{\mathrm{sat}}=\left[\mathrm{T}_{\mathrm{co}} *\left(\mathrm{e}^{\mathrm{x}}\right)-\mathrm{T}_{\mathrm{ci}}\right] /\left(\mathrm{e}^{\mathrm{x}}-1\right)
\end{aligned}
$$


Table IV. Net Condenser Heat Load at Various Loading Conditions

\begin{tabular}{|c|c|c|c|c|c|c|c|c|c|c|c|c|}
\hline \multirow{2}{*}{ Parameters } & \multicolumn{12}{|c|}{ Load (MW) } \\
\hline & 366 & 376 & 385 & 392 & 407 & 421 & 441 & 449 & 484 & 518 & 521 & 524 \\
\hline $\begin{array}{l}\text { MS Flow } \\
(\mathrm{kg} / \mathrm{s})\end{array}$ & 298 & 313 & 311 & 328 & 339 & 348 & 368 & 375 & 399 & 429 & 429 & 435 \\
\hline $\begin{array}{l}\text { MS press } \\
\text { (bar) }\end{array}$ & 129 & 159 & 131 & 168 & 161 & 155 & 161 & 166 & 165 & 163 & 165 & 165 \\
\hline $\begin{array}{l}\text { MS temp } \\
\left({ }^{\circ} \mathrm{C}\right)\end{array}$ & 540 & 539 & 539 & 538 & 539 & 538 & 536 & 537 & 538 & 535 & 538 & 536 \\
\hline $\begin{array}{l}\text { RH flow } \\
(\mathrm{kg} / \mathrm{s})\end{array}$ & 274 & 287 & 286 & 300 & 310 & 319 & 335 & 342 & 363 & 389 & 388 & 392 \\
\hline $\begin{array}{l}\text { HRH press } \\
\text { (bar) }\end{array}$ & 29 & 30 & 31 & 31 & 33 & 33 & 35 & 36 & 38 & 41 & 41 & 41 \\
\hline $\begin{array}{l}\text { HRH temp } \\
\left({ }^{\circ} \mathrm{C}\right)\end{array}$ & 538 & 536 & 541 & 532 & 535 & 540 & 527 & 531 & 536 & 538 & 540 & 539 \\
\hline $\begin{array}{l}\text { CRH press } \\
\text { (bar) }\end{array}$ & 31 & 33 & 33 & 34 & 35 & 36 & 38 & 39 & 41 & 44 & 44 & 44 \\
\hline $\begin{array}{l}\text { CRH temp } \\
\left({ }^{\circ} \mathrm{C}\right)\end{array}$ & 341 & 331 & 341 & 328 & 332 & 334 & 333 & 332 & 336 & 337 & 339 & 338 \\
\hline $\begin{array}{l}\text { FW flow } \\
(\mathrm{kg} / \mathrm{s})\end{array}$ & 266 & 291 & 285 & 308 & 317 & 331 & 352 & 361 & 378 & 411 & 410 & 425 \\
\hline $\begin{array}{l}\text { Final FW } \\
\text { press after } \\
\text { HP heaters } \\
\text { (bar) }\end{array}$ & 149 & 177 & 151 & 186 & 181 & 177 & 184 & 189 & 191 & 192 & 193 & 194 \\
\hline $\begin{array}{l}\text { Final FW } \\
\text { temp at } \\
\text { ECO inlet } \\
\left({ }^{\circ} \mathrm{C}\right)\end{array}$ & 237 & 240 & 240 & 241 & 243 & 244 & 247 & 248 & 251 & 255 & 255 & 256 \\
\hline $\begin{array}{l}\text { TDBFP } \\
\text { FW } \\
\text { suction } \\
\text { press (bar) }\end{array}$ & 5.9 & 6.0 & 6.1 & 6.2 & 6.3 & 6.5 & 6.7 & 6.8 & 7.2 & 7.6 & 7.7 & 7.7 \\
\hline $\begin{array}{l}\text { TDBFP } \\
\text { FW } \\
\text { suction } \\
\text { temp }\left({ }^{\circ} \mathrm{C}\right)\end{array}$ & 157 & 158 & 159 & 159 & 160 & 161 & 163 & 163 & 165 & 168 & 168 & 168 \\
\hline $\begin{array}{l}\text { TDBFP } \\
\text { FW } \\
\text { discharge } \\
\text { press (bar) }\end{array}$ & 151 & 179 & 154 & 189 & 183 & 180 & 187 & 193 & 195 & 196 & 198 & 199 \\
\hline $\begin{array}{l}\text { TDBFP } \\
\text { FW } \\
\text { discharge } \\
\text { temp }\left({ }^{\circ} \mathrm{C}\right)\end{array}$ & 156 & 158 & 158 & 160 & 161 & 162 & 164 & 164 & 167 & 170 & 170 & 170 \\
\hline $\begin{array}{l}\text { RH spray } \\
(\mathrm{kg} / \mathrm{s})\end{array}$ & 0 & 1 & 1 & 0 & 1 & 1 & 1 & 2 & 1 & 2 & 1 & 1 \\
\hline $\begin{array}{l}\text { RH spray } \\
\text { press bar) }\end{array}$ & 83 & 85 & 87 & 89 & 92 & 95 & 100 & 102 & 110 & 117 & 118 & 119 \\
\hline $\begin{array}{l}\text { RH spray } \\
\text { temp }\left({ }^{\circ} \mathrm{C}\right)\end{array}$ & 156 & 158 & 158 & 160 & 161 & 162 & 164 & 164 & 167 & 170 & 170 & 170 \\
\hline $\begin{array}{l}\text { SH spray } \\
(\mathrm{kg} / \mathrm{s})\end{array}$ & 32 & 22 & 26 & 19 & 22 & 17 & 16 & 14 & 22 & 19 & 19 & 11 \\
\hline $\begin{array}{l}\text { SH spray } \\
\text { press (bar) }\end{array}$ & 147 & 174 & 150 & 183 & 178 & 175 & 181 & 186 & 188 & 189 & 191 & 192 \\
\hline $\begin{array}{l}\text { SH spray } \\
\text { temp }\left({ }^{\circ} \mathrm{C}\right)\end{array}$ & 237 & 240 & 240 & 241 & 243 & 244 & 247 & 248 & 251 & 255 & 255 & 256 \\
\hline $\begin{array}{l}\text { Voltage } \\
(\mathrm{kV})\end{array}$ & 21 & 21 & 22 & 22 & 22 & 21 & 22 & 22 & 21 & 22 & 22 & 22 \\
\hline
\end{tabular}




\begin{tabular}{|c|c|c|c|c|c|c|c|c|c|c|c|c|}
\hline $\begin{array}{l}\text { Power } \\
\text { factor }\end{array}$ & 0.680 & 0.990 & 0.940 & 1.010 & 0.700 & 0.900 & 0.980 & 0.650 & 1.010 & 0.970 & 0.970 & 0.960 \\
\hline $\begin{array}{l}\mathrm{CW} \text { inlet } \\
\text { average } \\
\left({ }^{\circ} \mathrm{C}\right)\end{array}$ & 29 & 30 & 30 & 31 & 31 & 30 & 30 & 31 & 30 & 30 & 30 & 30 \\
\hline $\begin{array}{l}\mathrm{CW} \text { outlet } \\
\text { average } \\
\left({ }^{\circ} \mathrm{C}\right)\end{array}$ & 37 & 38 & 37 & 38 & 38 & 39 & 38 & 39 & 38 & 39 & 39 & 39 \\
\hline $\begin{array}{l}\text { Condenser } \\
\text { heat load } \\
(\mathrm{kJ} / \mathrm{s})\end{array}$ & 552543 & 556030 & 558019 & 569038 & 592434 & 595945 & 612090 & 620618 & 664916 & 701733 & 698874 & 689430 \\
\hline
\end{tabular}

Table V. Break-up of Deviation of Actual and Calculated Condenser Pressure

\begin{tabular}{|c|c|c|c|c|c|c|c|c|c|c|c|c|}
\hline \multirow{2}{*}{ Parameters } & \multicolumn{12}{|c|}{ Load (MW) } \\
\hline & 366 & 376 & 385 & 392 & 407 & 421 & 441 & 449 & 484 & 518 & 521 & 524 \\
\hline $\begin{array}{l}\text { Observed } \\
\text { Condenser } \\
\text { pressure (bar) }\end{array}$ & 0.088 & 0.102 & 0.093 & 0.098 & 0.100 & 0.098 & 0.097 & 0.106 & 0.099 & 0.102 & 0.102 & 0.102 \\
\hline Actual $\Delta \mathrm{T}\left({ }^{\circ} \mathrm{C}\right)$ & 7.4 & 7.7 & 7.9 & 7.4 & 7.6 & 9.0 & 7.9 & 8.2 & 8.2 & 8.6 & 8.6 & 8.7 \\
\hline $\begin{array}{l}\text { Inlet cooling } \\
\text { water } \\
\text { temperature } \\
\text { correction factor }\end{array}$ & 1.062 & 1.065 & 1.063 & 1.069 & 1.069 & 1.065 & 1.065 & 1.070 & 1.065 & 1.066 & 1.065 & 1.066 \\
\hline $\begin{array}{l}\text { Corrected Heat } \\
\text { transfer } \\
\text { coefficient } \\
\left(\mathrm{KW} / \mathrm{m}^{2}-{ }^{\circ} \mathrm{C}\right)\end{array}$ & 3.58 & 3.59 & 3.59 & 3.61 & 3.61 & 3.60 & 3.60 & 3.61 & 3.59 & 3.60 & 3.59 & 3.60 \\
\hline $\operatorname{LMTD}\left({ }^{\circ} \mathrm{C}\right)$ & 7.5 & 7.5 & 7.5 & 7.6 & 8.0 & 8.0 & 8.3 & 8.3 & 9.0 & 9.5 & 9.4 & 9.3 \\
\hline $\begin{array}{l}\text { Saturation } \\
\text { temperature }\left({ }^{\circ} \mathrm{C}\right)\end{array}$ & 41.2 & 42.0 & 41.7 & 42.6 & 43.1 & 43.4 & 42.9 & 44.0 & 43.5 & 44.5 & 44.3 & 44.5 \\
\hline $\begin{array}{l}\text { Calculated/targe } \\
\mathrm{t} \text { condenser } \\
\text { pressure (bar) }\end{array}$ & 0.079 & 0.082 & 0.081 & 0.085 & 0.087 & 0.088 & 0.086 & 0.091 & 0.089 & 0.093 & 0.093 & 0.094 \\
\hline $\begin{array}{l}\text { Theoretical } \Delta \mathrm{T} \\
\left({ }^{\circ} \mathrm{C}\right)\end{array}$ & 5.8 & 5.8 & 5.8 & 5.9 & 6.2 & 6.2 & 6.4 & 6.5 & 6.9 & 7.3 & 7.3 & 7.2 \\
\hline $\begin{array}{l}\text { Theoretical TTD } \\
\left({ }^{\circ} \mathrm{C}\right)\end{array}$ & 3.4 & 4.2 & 3.9 & 4.6 & 4.9 & 5.2 & 4.5 & 5.6 & 4.6 & 5.2 & 5.0 & 5.3 \\
\hline $\begin{array}{l}\text { B.P due to } \mathrm{CW} \\
\text { inlet temperature } \\
\text { (bar) }\end{array}$ & 0.068 & 0.074 & 0.071 & 0.079 & 0.081 & 0.080 & 0.077 & 0.087 & 0.080 & 0.085 & 0.083 & 0.085 \\
\hline
\end{tabular}




\begin{tabular}{|c|c|c|c|c|c|c|c|c|c|c|c|c|}
\hline $\begin{array}{l}\text { B.P due to CW } \\
\text { flow and CW } \\
\text { inlet temperature } \\
\text { (bar) }\end{array}$ & 0.075 & 0.081 & 0.079 & 0.085 & 0.087 & 0.092 & 0.084 & 0.095 & 0.085 & 0.090 & 0.089 & 0.092 \\
\hline $\begin{array}{l}\text { Variation due to } \\
\mathrm{CW} \text { inlet } \\
\text { temperature } \\
\text { (bar) }\end{array}$ & -0.010 & -0.008 & -0.010 & -0.006 & -0.006 & -0.009 & -0.009 & -0.005 & -0.009 & -0.009 & -0.009 & -0.008 \\
\hline $\begin{array}{l}\text { Variation due to } \\
\text { CW flow (bar) }\end{array}$ & 0.006 & 0.008 & 0.008 & 0.006 & 0.006 & 0.013 & 0.007 & 0.008 & 0.005 & 0.006 & 0.006 & 0.007 \\
\hline $\begin{array}{l}\text { Variation due to } \\
\text { air/dirty tubes } \\
\text { (bar) }\end{array}$ & 0.014 & 0.021 & 0.014 & 0.013 & 0.013 & 0.006 & 0.013 & 0.011 & 0.014 & 0.012 & 0.013 & 0.010 \\
\hline
\end{tabular}

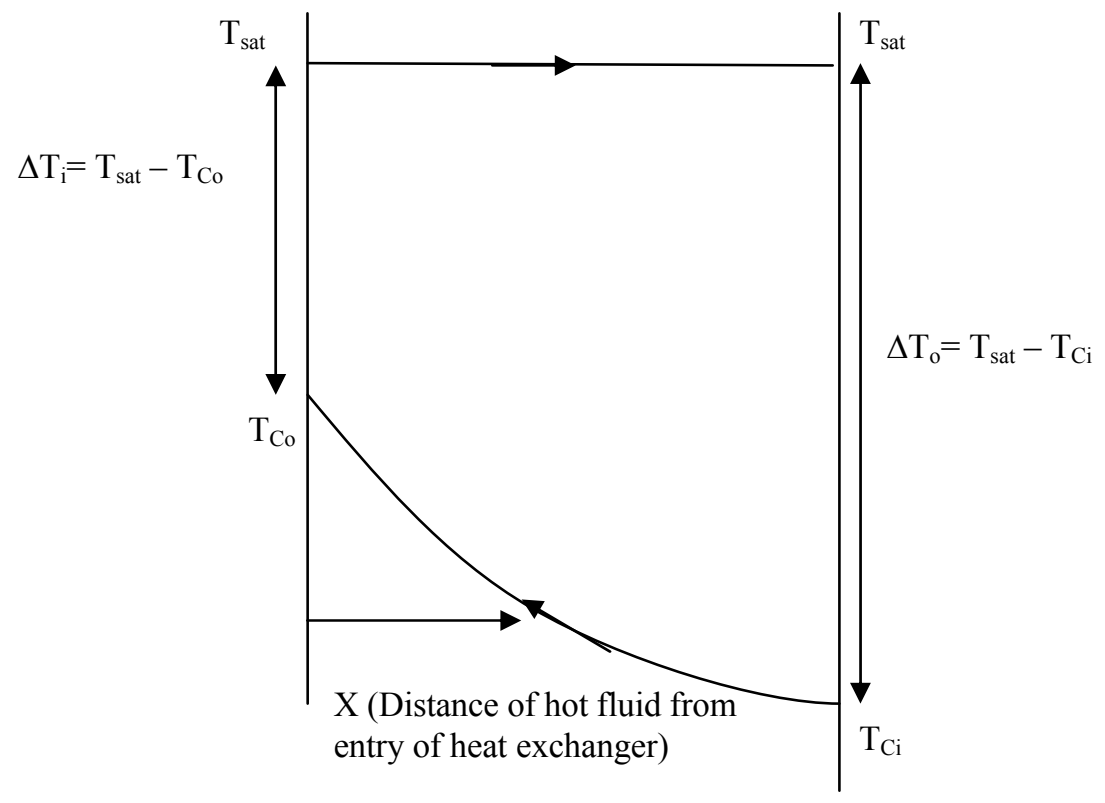

Fig. 2 Temperature profiles of hot and cold fluids

Saturation pressure $\left(\mathrm{P}_{\text {sat }}\right)$ corresponding to saturation temperature $\left(\mathrm{T}_{\text {sat }}\right)$ of steam is termed as calculated/target condenser back pressure. Calculated/target condenser pressure at different loading conditions is shown in Table V.

\section{IV.RESULT AND DISCUSSIONS}

The Deviation of actual condenser pressure from calculated/target condenser pressure is shown in Fig. 4. This deviation is attributed to three main factors - (1) variation due to cooling water inlet temperature and variation due to cooling water flow as shown in Fig. 3 and (2) variation due to air ingress/dirty tubes as shown in Fig.4.

From the results it can be concluded that condenser back pressure improves due to drop in $\mathrm{CW}$ inlet temperature when compared to design $\mathrm{CW}$ inlet temperature. Variation in condenser back pressure due to deviation in $\mathrm{CW}$ inlet temperature will be considered as negative whenever cooling water inlet temperature would be less than the design cooling water inlet temperature. Significant deviation in condenser back pressure is observed due to lower CW flow than the design; causes for lower CW flow may be poor performance of CW pumps or due to low suction head.

More deviation in back pressure due to air ingress or dirty tubes is also observed. It may be due to actual air ingress which can be appropriately judged by observing hot well water temperature. It can be noticed that at 366 MW load and cooling water inlet temperature of 29 deg C, instead of 0.079 bar back pressure (calculated), the actual condenser pressure is 0.088 bar. This deviation ( 0.01 bar) between actual condenser back pressure and calculated condenser back pressure is attributed to three factors.

(1) Deviation due to $\mathrm{CW}$ inlet temperature is -0.010 bar, (a gain in back pressure), which implies that back pressure can be lowered by 0.010 bar had the cooling water inlet temperature was at $29 \mathrm{deg} \mathrm{C}$, by keeping other parameters constant. 
(2) Deviation due to $\mathrm{CW}$ flow is 0.006 bar, a loss in pressure which implies that $\mathrm{CW}$ flow is less than design flow, which is indicated by higher cooling water temperature rise through condenser tubes.

(3) Major deviation is due to air ingress/dirty tubes. Deviation due to air ingress/dirty tubes as an individual entity shows a 0.014 bar loss of pressure.

In case of air ingress, there are methods available for online air ingress testing. But among them, helium leakage is best as it can be performed online without affecting power generation.

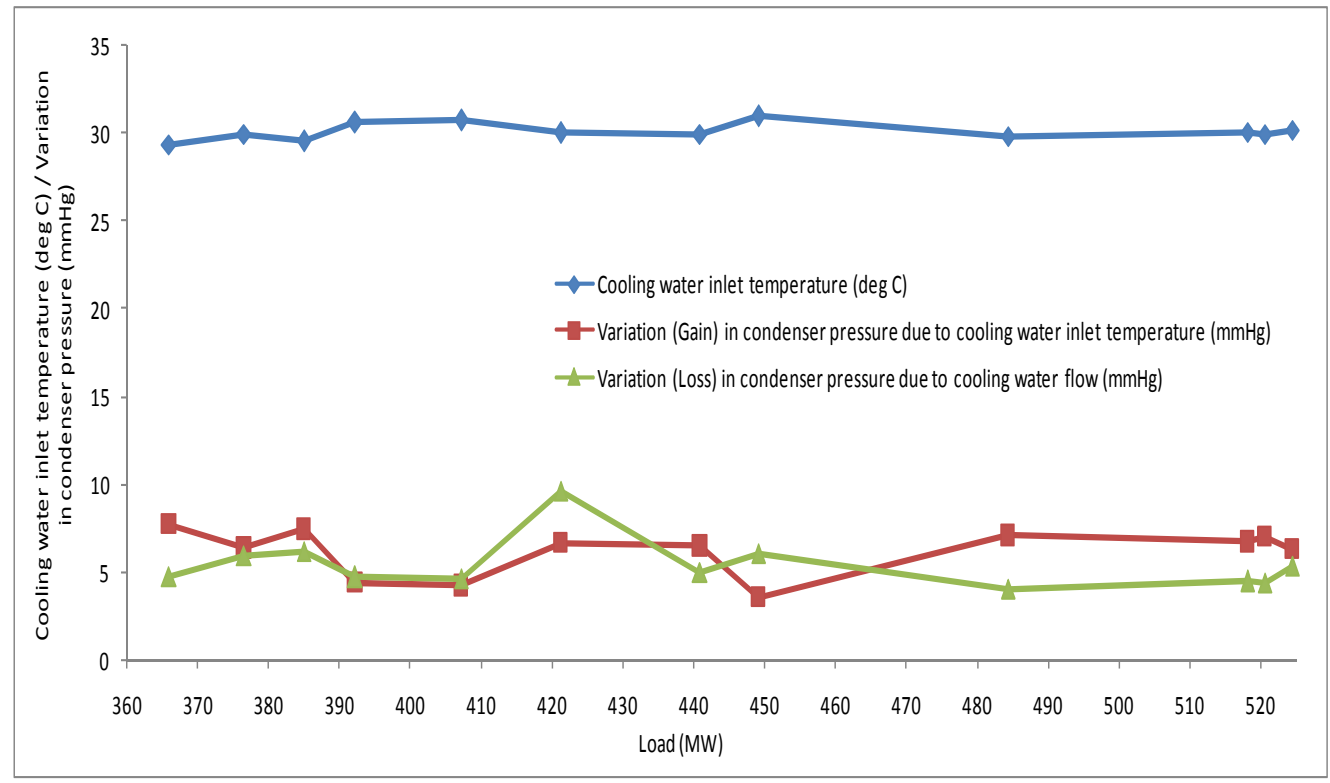

Fig. 3. Deviation of observed condenser pressure from calculated/target condenser pressure due to cooling water inlet temperature and cooling water flow.

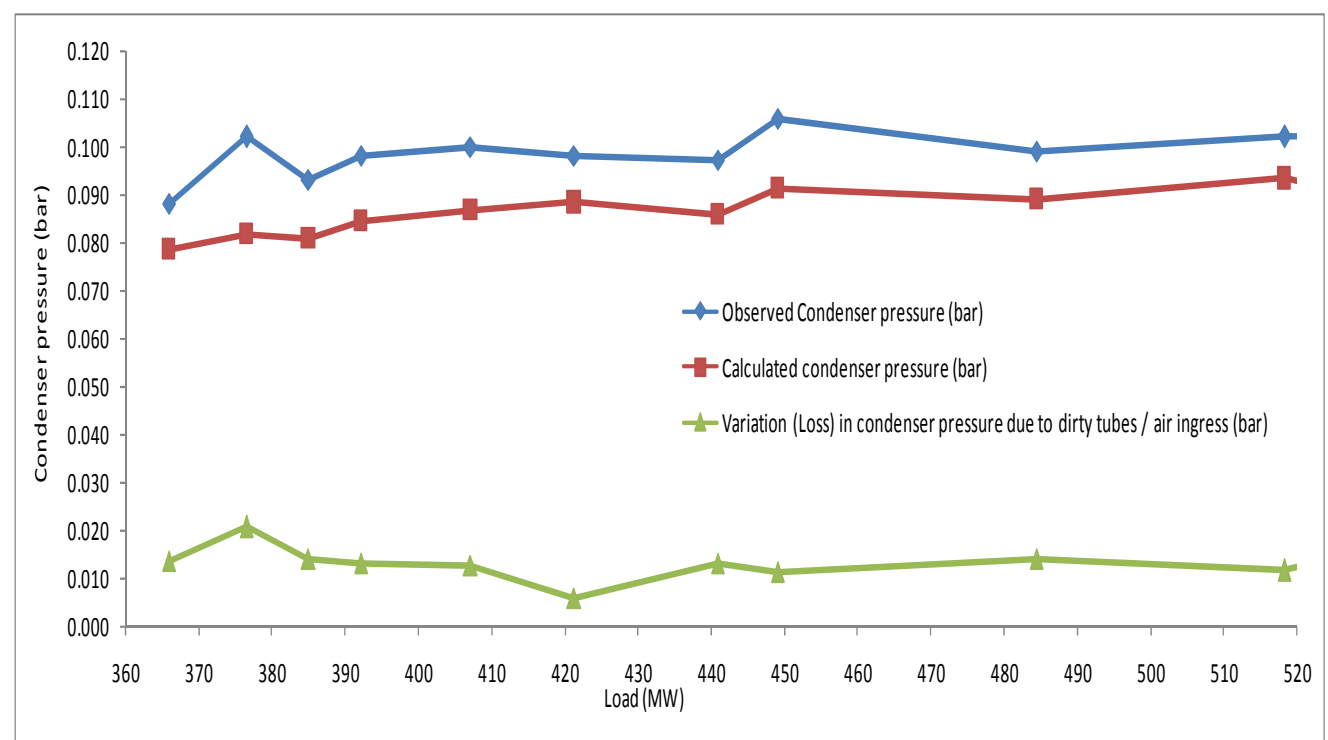

Fig. 4. Deviation of observed condenser pressure from calculated/target condenser pressure due to dirty tubes and air ingressing.

\section{ACKNOWLEDGMENT}

The author is grateful to the management and staff of HNPCL and SOMC for their cooperation and helpful discussions.

\section{REFERENCES}

[1] Ankur Geete and A.L. Khandwawala, "Thermodynamic analysis of 120MW thermal power plant with combined effect of constant inlet pressure (124.61bar) and different inlet temperatures", Elsevier, Case studies in Thermal Engineering 1 (2013) 17-25.

[2] Heat Exchange Institute incorporated standards for steam surface condensers, tenth edition. 
[3] J.P.Holman, Heat Transfer, McGraw-hill (ISBN-10:0-07-844785-2),Eight Edition.

[4] A.B.Gill, Power Plant Performance, Elsevier (ISBN-978-1-4831-0000-5), first edition.

[5] Vikram Haldkar, Abhay kumar sharma, R.K Ranjan, V.K Bajpai,"Parametric analysis of surface condenser for thermal power plant", IJTT, Vol.3, No.4, (Dec 2013).

[6] Rafal Laskowski," Relations for steam power plant condenser perfromance in off-design conditions in the function of inlet parameters and those relevant in reference conditions", Elsevier, Applied thermal engineering 103 (2016) 528-536.

[7] Sami I. Attia,"The influence of condenser cooling water temperature on the thermal efficiency of a nuclear power plant", Elsevier, Annals of nuclear energy (2015), http://dx.doi.org/10.1016/j.anucene.2015.02.023.

[8] R.K.Kapooria, S Kumar and K S kasana,”Technological investigations and efficiency analysis of a steam heat exchange condenser: conceptual design of a hybrid steam condenser",Journal of energy in Southern Africa, Vol. 19, No.3, August 2008.

[9] Ajeet Singh Sikarwar, Devendra Dandotiya, Surendra Kumar Agrawal,"Performance analysis of a surface condenser under various operating parameters", IJERA, Vol.3, Issue 4, Jul-Aug 2013, pp. 416-421.

[10] Amir vosough, Alireza falahat, Sadegh vosough, Hasan nasr esfehani, Azam behjat and Roya naseri rad, "Improvement Power plant efficiency with condenser pressure", IJMSE, Vol. 2, No.3, June 2011.

[11] Said M.A. Ibrahim and Sami I. Attia,"Influence of condenser cooling seawater salinity changes on the thermal performance of nuclear power plant", Elsevier, Progress in nuclear energy 79 (2015) 115-126. 